\title{
Influence of Different Patellofemoral Design Variations Based on Genesis II Total Knee Endoprosthesis on Patellofemoral Pressure and Kinematics
}

\author{
Ulf G. Leichtle, ${ }^{1}$ Barbara Lange, ${ }^{1}$ Yvonne Herzog, ${ }^{2}$ Peter Schnauffer, \\ Carmen I. Leichtle, ${ }^{1}$ Nikolaus Wülker, ${ }^{1}$ and Andrea Lorenz ${ }^{1}$ \\ ${ }^{1}$ Department of Orthopaedic Surgery, University Hospital Tübingen, 72076 Tübingen, Germany \\ ${ }^{2}$ Institute of Aircraft Design, University of Stuttgart, 70569 Stuttgart, Germany
}

Correspondence should be addressed to Ulf G. Leichtle; ulf.leichtle@med.uni-tuebingen.de

Received 16 October 2016; Accepted 5 January 2017; Published 31 January 2017

Academic Editor: Estefanía Peña

Copyright (C) 2017 Ulf G. Leichtle et al. This is an open access article distributed under the Creative Commons Attribution License, which permits unrestricted use, distribution, and reproduction in any medium, provided the original work is properly cited.

\begin{abstract}
In total knee arthroplasty (TKA), patellofemoral groove design varies greatly and likely has a distinct influence on patellofemoral biomechanics. To analyse the selective influence, five patellofemoral design variations were developed based on Genesis II total knee endoprosthesis (original design, being completely flat, being laterally elevated, being medially elevated, and both sides elevated) and made from polyamide using rapid prototyping. Muscle-loaded knee flexion was simulated on 10 human knee specimens using a custom-made knee simulator, measuring the patellofemoral pressure distribution and tibiofemoral and patellofemoral kinematics. The measurements were carried out in the native knee as well as after TKA with the 5 design prototypes. The overall influence of the different designs on the patellofemoral kinematics was small, but we found detectable effects for mediolateral tilt $(p<0.05$ for $35^{\circ}-80^{\circ}$ flexion) and translation of the patella $\left(p<0.045\right.$ for $20^{\circ}-65^{\circ}$ and $\left.75^{\circ}-90^{\circ}\right)$, especially for the completely flat design. Considering patellofemoral pressures, major interindividual differences were seen between the designs, which, on average, largely cancelled each other out. These results suggest that the elevation of the lateral margin of the patellofemoral groove is essential for providing mediolateral guidance, but smooth contouring as with original Genesis II design seems to be sufficient. The pronounced interindividual differences identify a need for more patellofemoral design options in TKA.
\end{abstract}

\section{Introduction}

Leslie Gordon Percival Shiers is considered a pioneer of modern knee endoprosthetics. In 1954, he published in the Journal of Bone and Joint Surgery (BR) encouraging findings involving a hinged prosthesis made of stainless steel, which at that time he had used in four patients. In the conclusion section of his original publication there is interesting assertion he made that illustrates how far knee endoprosthetics have come since then: "However, few surgeons will ever see fifty patients requiring arthroplasty of the knee, let alone operate on them, even in five years" [1].

Shiers would be surprised to see that in the meantime there are many surgeons who perform total knee arthroplasty on more than 100 patients a year and approximately 130.000 patients per year are operated on in Germany alone [2].
With meanwhile very good clinical results, persisting anterior knee pain, however, continues to be an unsolved problem [36]. The reasons are multifactorial. Among other things, in this context the indication for implantation of an additional patellar resurfacing continues to be a controversial subject of debate, whereby current meta-analyses rather favour the performance of patellar resurfacing $[3,7]$. Correct positioning of the femoral and tibial components of the prosthesis in terms of rotation $[8,9]$, correction of the leg axis, reconstruction of the joint line [10], careful soft tissue balancing, and avoiding overstuffing with implants that are too big [11] are other important influencing factors the surgeon needs to consider. Another factor often which is paid little attention to and that likely has a strong influence on the outcome is the design of the implanted endoprosthesis [12-17]. When comparing prosthesis types of various manufacturers, one sees that the 
designs sometimes differ dramatically in terms of the contour of the femoral groove. Among others, laterally raised and deeply recessed variations exist in addition to relatively flat designs.

The outcome after implantation of different prosthesis systems of various manufacturers has been compared in numerous clinical studies [12, 15, 17, 18]. Among other things, a significant influence of the design on the postoperative, patellofemoral complication rate [17], the risk and location of osteoscleroses [18], and the necessity for intraoperative lateral retinacular release [12] was found in those studies.

When comparing Genesis II and the Vanguard prosthesis, which differ considerably with regard to the design of the patellofemoral groove, no significant difference was found in terms of the clinical scores and radiological parameters evaluated [15]. In these studies, however, only the position of the patella during a static snapshot was assessed. In contrast, in intraoperative measurements performed on two prostheses differing only in terms of patellofemoral groove design [19], a considerable difference in the position of the patella was found, albeit only in unloaded state. But because of the multifactorial influences mentioned, it is next to impossible to selectively extrapolate the difference of differing groove designs in clinical studies.

On the other hand, biomechanical in vitro studies make it possible to objectively measure patellar kinematics and patellofemoral pressure distribution while simulating muscle-loaded knee bends. Due to the great differences in the experimental settings, however, it is not constructive here as well to compare the numerous results about patellar kinematics [11, 20-22] or patellofemoral pressure [23-28] from various studies on different prosthesis designs. But it is also difficult in studies with a standardised setting to extrapolate the influence of different patellofemoral groove designs, to the extent as compared in prosthesis systems of various manufacturers $[16,29,30]$, as they differ not only in terms of the shape of the patellofemoral groove but also in the overall design. The altogether strong influence of design on patellofemoral pressure distribution $[16,29,30]$ as well as specifically the mediolateral shifting and tilting of the patella [16] was able to be proven in these studies, however.

Initial in vitro studies using two prosthesis designs differing only in terms of patellofemoral groove design were conducted back in the early 90 s by Yoshii et al. and confirmed the importance of the patellofemoral groove design. The specific influence of the trochlear angle $\left(0^{\circ}\right.$ and/or $\left.7^{\circ}\right)$ has already been evaluated several times [13,31], with differing results. However, the great variation of the patellofemoral groove design among the individual prosthesis manufacturers underscores the necessity for further biomechanical studies in this area.

The purpose of this in vitro biomechanical study was therefore to design prototypes with different patellofemoral groove contouring using the same basic design of a commonly used standard endoprosthesis (Genesis II) and to investigate its influence on patellofemoral kinematics and pressures during a muscle-loaded knee bend, in order to selectively extrapolate the influence of different design variations in the area of the patellofemoral groove of total knee

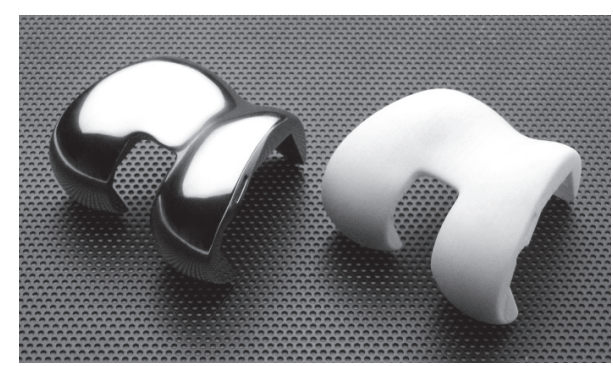

FIGURE 1: Left: original implant made from metal; right: "copy" made from polyamide, created by means of parameterised CAD modelling and rapid prototyping.

endoprostheses. To do this, muscle-loaded knee bends using human knee specimens should be simulated using a complex knee simulator. The null hypothesis was that patellofemoral groove design had no effect on patellar kinematics and patellofemoral pressure.

\section{Materials and Methods}

As part of a cooperative project with the Institute of Aircraft Design of the University of Stuttgart, three knee endoprostheses of different manufacturers were scanned in using a two-camera system (ATOS, GOM) and the data sets were converted into STL data (Standard Tessellation Language using special software, a file format for depicting physical surfaces using small triangles). Associatively parameterised CAD models were created based on that data using the CAD software CATIA [32]. They are characterized by the fact that through simple parameter variations the models of all manufacturers could be imaged and new implant designs created. Copies of the original implant or also modified prototypes could then be made ("printed") directly from synthetic material or metal using the laser sintering method. The surface of the prototypes was slightly rough and microporous immediately after being made and had to be polished on the sliding surface with fine pored abrasive paper, in order to guarantee sufficient sliding ability. For this reason, before printing, the CAD models were provided with small allowance. The parametrised models of the original implants exhibited a high degree of conformity with the real parts scanned; the difference from the real geometry was a few $\mu \mathrm{m}$ here. Macroscopically, the original implant and "copy" made from polyamide were identical in shape (Figure 1).

In a preliminary work step, the true-to-original copies of Genesis II total knee endoprosthesis made from polyamide based on the parameterised CAD model were validated by means of double measurements and comparative measurements with the original implants [33].

Five groove designs were then devised and implant prototypes were made using this associatively parametrised CAD model (Figure 2):

(1) Original Genesis II design

(2) Completely flat patellofemoral groove 


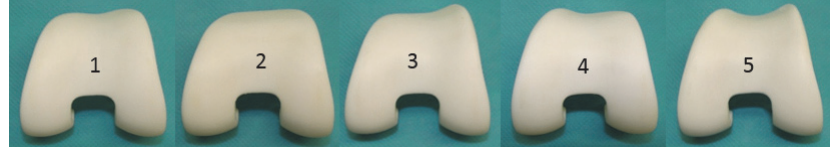

FIGURE 2: Design variations of Genesis II endoprosthesis with modified patellofemoral groove: (1) original design, (2) being flat, (3) being medially high, (4) being laterally high, and (5) both sides high.

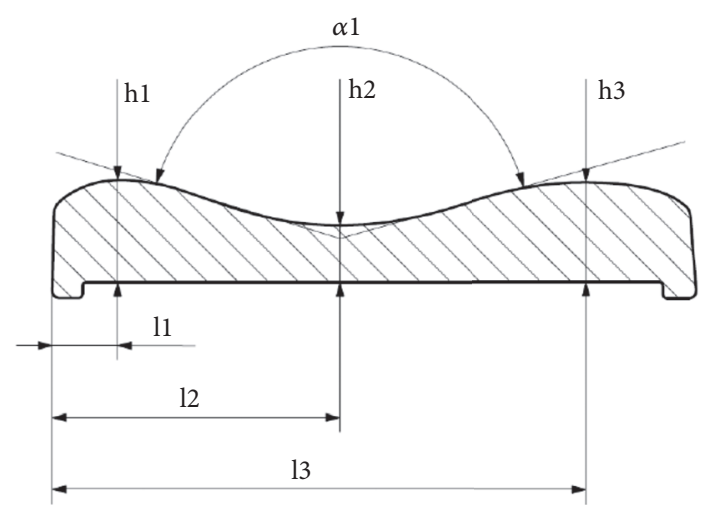

Figure 3: Parameters of the patellofemoral groove (from [32] with permission of the author).

(3) Groove medially raised by $2 \mathrm{~mm}$ compared to the original Genesis II groove and laterally lowered by $1 \mathrm{~mm}$

(4) Groove laterally raised by $2 \mathrm{~mm}$ compared to the original Genesis II groove and medially lowered by $1 \mathrm{~mm}$

(5) Groove medially and laterally raised by $3 \mathrm{~mm}$ compared to the original Genesis II groove

When modifying the design, only the heights of the two groove margins were adjusted $(\mathrm{h} 1, \mathrm{~h} 3)$ and their angles altered as a result as well $(\alpha 1)$ (Figure 3$)$. The minimum height in the area of the groove (facies patellaris, h2) as well as the lateral distance of the margins $(11,13)$ and of the facies patellaris (12) was left at the original dimensions.

For evaluating the groove designs, an in vitro study was conducted on ten fresh frozen human knee specimens on the knee simulator of the Orthopaedic University Hospital Tübingen. The specially designed knee simulator, already well-established through its use in numerous studies [25, 3436], makes it possible to simulate muscle-loaded knee bends in the $15^{\circ}-90^{\circ}$ flexion range. During the flexion motion, forces are applied onto three quadriceps muscles (M. vastus lateralis, $M$. rectus femoris, and M. vastus medialis) and two hamstrings (M. semimembranosus and M. biceps femoris) by means of servomotors. While the forces on the hamstrings are kept constant, the quadriceps forces are set such that the vertical reaction force on the ankle remains constant [35]. In this study, $50 \mathrm{~N}$ ankle force was simulated. During the simulation of the muscle-loaded knee bend, the kinematics of the tibia, femur, and patella were measured using an ultrasonic motion analysis system (CMS-H, Zebris, Isny, Germany; resolution: $0.085 \mathrm{~mm}$, accuracy: $1 \mathrm{~mm}$ ). Additionally, the patellofemoral pressure distribution was recorded using resistive pressure measuring foils (K-Scan, TekScan, Boston, MA, USA). A reference measurement was performed at the start of each measurement series in order to determine the segmental coordinate systems [34].

Three different muscle force distributions on the quadriceps muscle were tested:

(1) Central (symmetrical) force distribution on the quadriceps muscle (33\% vastus lateralis; $34 \%$ rectus femoris; $33 \%$ vastus medialis)

(2) Mainly lateral force transmission (67\% vastus lateralis; $33 \%$ rectus femoris; $0 \%$ vastus medialis)

(3) Mainly medial force transmission ( $0 \%$ vastus lateralis; $33 \%$ rectus femoris; $67 \%$ vastus medialis)

Each of the following knee conditions was completed with each of the three muscle force distributions on the quadriceps muscle, whereby two measurement cycles were recorded with the central force distribution:

(1) Native knee joint after arthrotomy

(2) Knee joint after implantation of five different prototypes in a randomised sequence

(3) Knee joint after implantation of five different prototypes in a randomised sequence and additional patellar resurfacing

The measured data were analysed based on the flexion angle in $1^{\circ}$ increments and, if applicable, averaged over the two repeated measures.

In order to exclude the influence of the various specimens, a repeated measures analysis of variance (ANOVA) was done for the different prosthesis designs and for the three muscle distribution patterns, selecting a significance level of $5 \%(\alpha=0.05)$. The calculation was done using MATLAB (anova_rm.m, MATLAB File Exchange). Post hoc $t$-tests were done using the Bonferroni correction method to determine the deviations of the individual prosthesis types from the native knee.

In addition to the mean values, the standard deviations (SD) were put in the diagrams as error bars at $5^{\circ}$ increments. The statistical significance in the ANOVA was also calculated and shown at those intervals.

\section{Results}

On average, the different designs had surprising little influence on patellofemoral kinematics. The various groove designs had no influence on the average alignment of the patella in craniocaudal and anteroposterior orientation. There was a detectable effect of the different design variations only on the mediolateral tilt $\left(p<0.05\right.$ between $35^{\circ}$ and $80^{\circ}$ flexion) and translation of the patella $\left(p<0.045\right.$ of $20^{\circ}-65^{\circ}$ and $75^{\circ}-90^{\circ}$ ) (Figure 4 ).

The lateral tilting of the patella was somewhat less with the laterally elevated and with both sides elevated design 

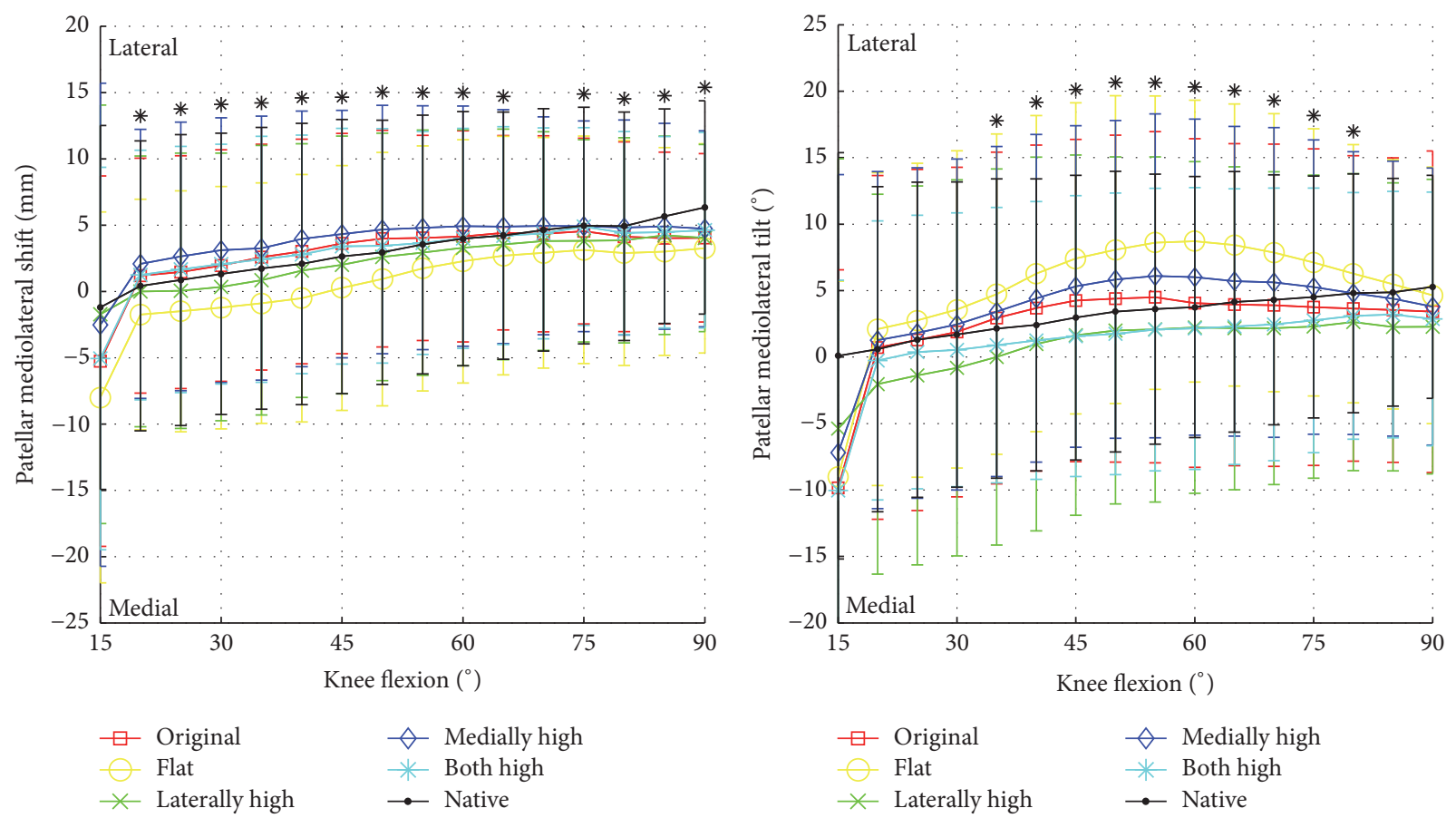

FIGURE 4: Patellofemoral kinematics with central muscle load after implantation of the different designs without patellar resurfacing (native, original, flat, laterally high, medially high, and both sides high) ( ${ }^{*}$ significant difference in ANOVA).

than in the native state and slightly higher with the medially elevated and completely flat design. Except for the completely flat design, however, where a lateral tilt of up to $5^{\circ}$ was measured, which between $45^{\circ}$ and $65^{\circ}(p<0.004)$ was also significant, the deviation from the native state was in the range of no more than $1^{\circ}-2^{\circ}$. For the mediolateral shift the deviations were likewise very minor $(1 \mathrm{~mm}-3 \mathrm{~mm})$. An interesting observation, however, was that the patella diverted medially in the case of the completely flat design, contrary to the tendency with the other designs. The difference with the native patella, however, was at maximum $3 \mathrm{~mm}$ not significant in any area here.

The additional performance of patellar resurfacing had no apparent influence on the patellofemoral kinematics.

Comparing the different design variations for patellofemoral pressure distribution, the effects of the five designs could often be better interpreted by observing each of the individual knee specimens separately. Large interindividual differences were determined between the ten knee specimens with respect to advantages and disadvantages of the respective design. Considering the maximum pressure averaged between $15^{\circ}$ and $50^{\circ}$, the lowest pressures could be achieved with each design, in the case of at least two of the ten specimens, except for the flat design. The highest pressures were achieved with the laterally and both sides elevated design in two specimens, however; the remaining six specimens showed maximum pressures with the flat design (Table 1).

The largest contact area of the patella was usually seen on the native knee joint, followed by original Genesis II design or the medially elevated design. The flat design had a smaller central contact area, which was located approximately in the area of the patellar ridge, whereas the contact area in the case of the deeply recessed design shifted more onto the condyles (Figure 5). After additional patellar resurfacing, the contact area decreased considerably in all design variations. In this case, the very selective contact was again central in the groove in the flat design and in the original as well as the deeply recessed design it was located laterally or medially in the area of the condyles (Figure 5).

When examining the total sample, many of the sometimes contradictory effects observed on the individual specimen averaged out. After implantation of the different designs, initially without patellar resurfacing, an overall considerable increase of the peak pressures measured was documented with a reduction of the patellofemoral contact area. While the original, medially elevated, laterally elevated, and both sides elevated (deeply recessed) designs produced similar results, the completely flat design had the highest peak pressures, especially at low flexion angles up to approximately $50^{\circ}$ (Figure 6).

An interesting observation was the influence of the various muscle loads on the displacement of the centre of pressure. In the case of the native knee, the influence of the asymmetrical muscle loads was the greatest; on the other hand, in the case of the flat groove design, hardly any effect was detectable (Figure 7).

Considering the situation after implantation of an additional patellar resurfacing, in all design variations there was a considerable further reduction in the contact area as well as a significant increase in the peak pressures measured. While most of the designs exhibited an almost identical pressure 

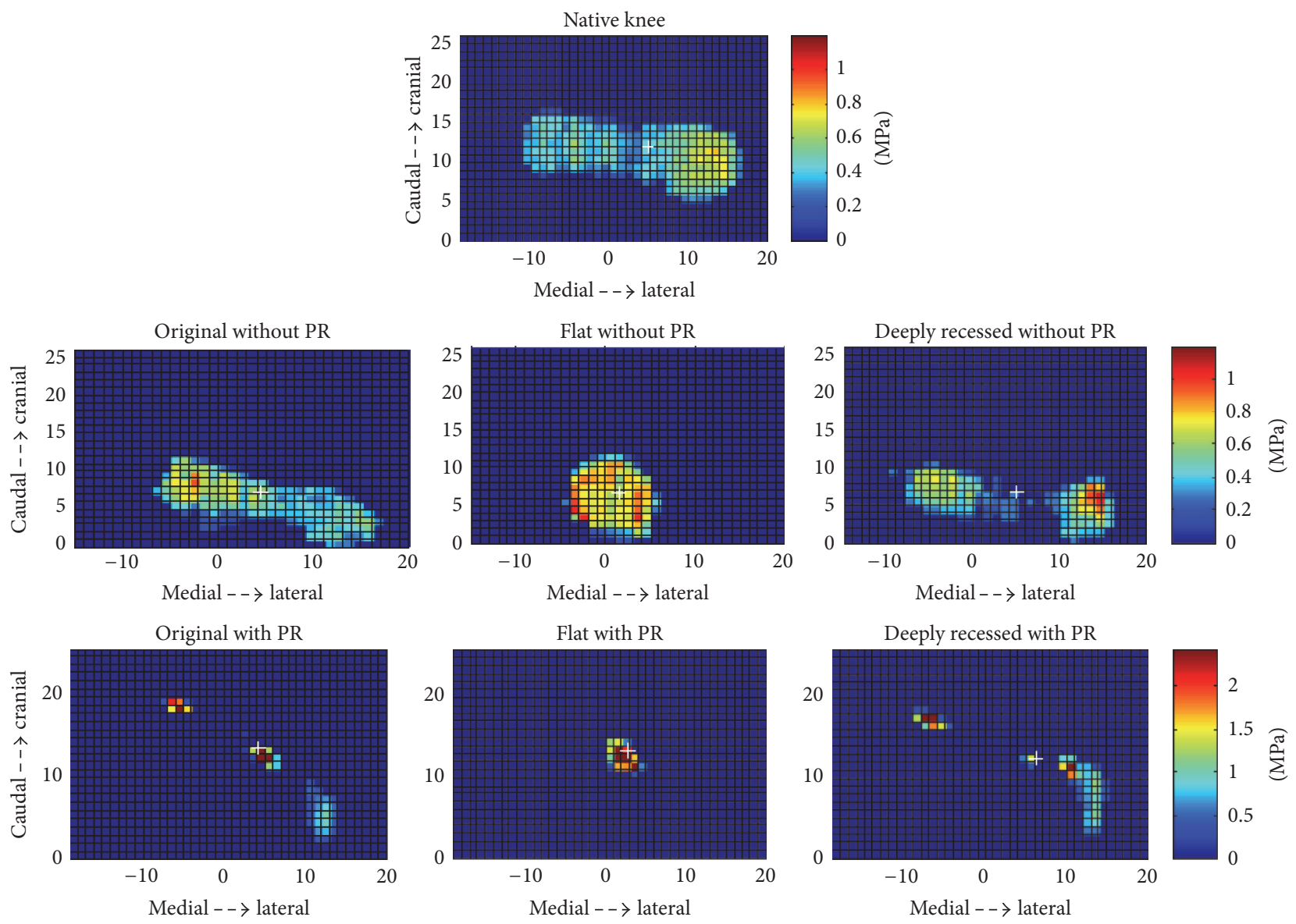

FIGURE 5: Example of pressure profile for the designs: native knee, original Genesis II design, flat design, and both sides elevated (deeply recessed), respectively, with and without additional patellar resurfacing (PR).

TABLE 1: Sequence of the five designs for observing maximum pressures for all ten measured knee specimens. Data is averaged over the flexion range of $15^{\circ}-50^{\circ}$, scaling ranges from $1=$ lowest pressure to $5=$ greatest pressure. The major interindividual differences as well as the poor performance of the flat design are clearly evident.

\begin{tabular}{|c|c|c|c|c|c|}
\hline & Original & Flat & Laterally high & Medially high & Both high \\
\hline Knee 1 & 2 & 5 & 4 & 3 & 1 \\
\hline Knee 2 & 4 & 5 & 3 & 2 & 1 \\
\hline Knee 3 & 2 & 5 & 4 & 3 & 1 \\
\hline Knee 4 & 4 & 3 & 1 & 2 & 5 \\
\hline Knee 5 & 1 & 5 & 2 & 4 & 3 \\
\hline Knee 6 & 2 & 4 & 5 & 1 & 3 \\
\hline Knee 7 & 3 & 4 & 5 & 1 & 2 \\
\hline Knee 8 & 4 & 5 & 3 & 1 & 2 \\
\hline Knee 9 & 1 & 5 & 4 & 2 & 3 \\
\hline Knee 10 & 2 & 4 & 1 & 3 & 5 \\
\hline Mean & 2.5 & 4.5 & 3.2 & 2.2 & 2.6 \\
\hline
\end{tabular}

gradient, the curve of the flat groove design again attracted the attention. Especially with increased lateral muscle load, considerably higher maximum pressures were documented in the flexion range between approximately $40^{\circ}$ and $80^{\circ}$, compared to the other designs (Figure 8).

\section{Discussion}

Considering the four design variations modified in terms of the patellofemoral groove as well as the original design of Genesis II endoprosthesis, we were able to show that 

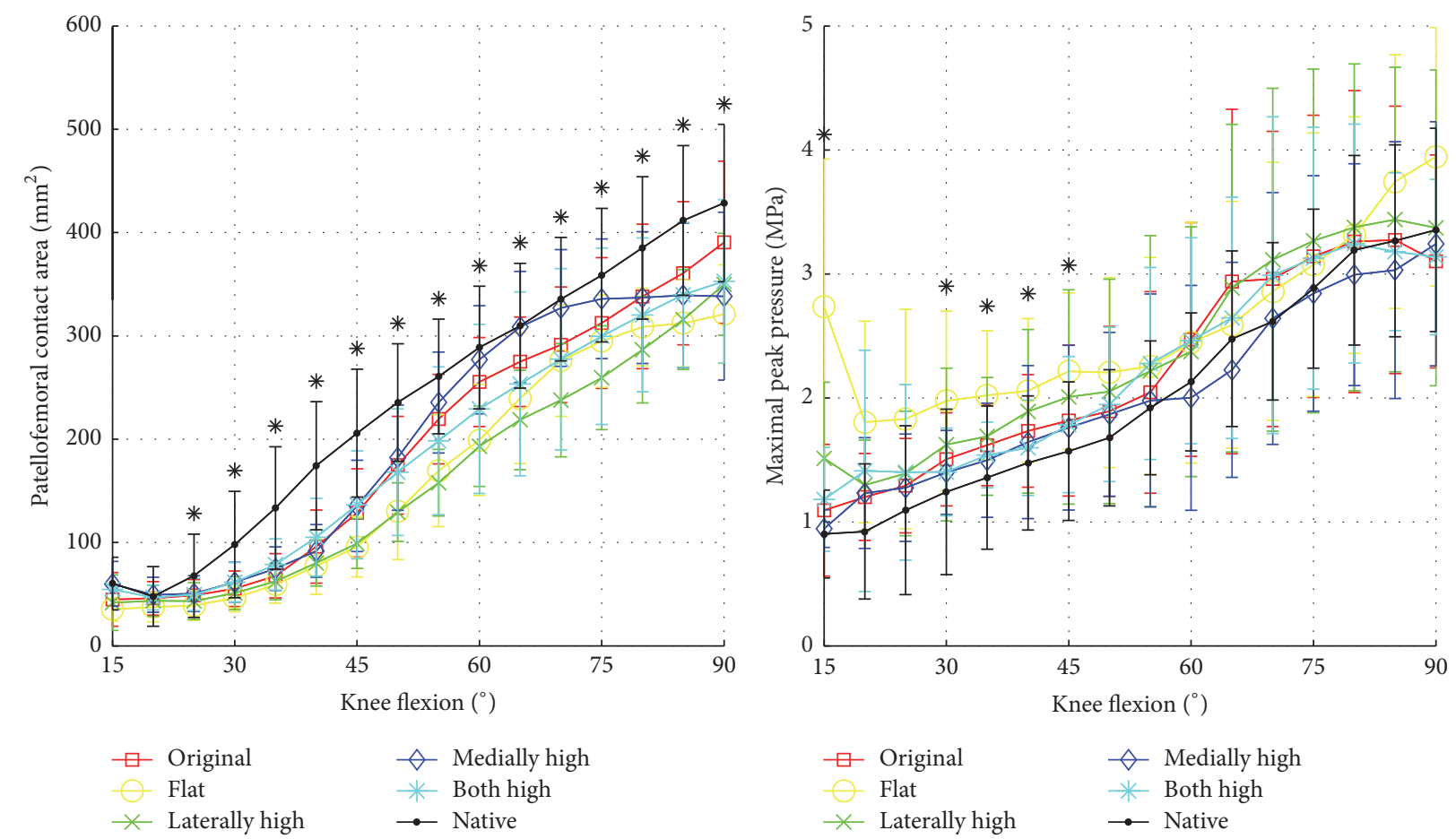

FIGURE 6: Patellofemoral contact area and maximum pressures with central muscle load after implantation of the different design variations without patellar resurfacing (native, original, flat, laterally high, medially high, and both high) (* significant difference in ANOVA).
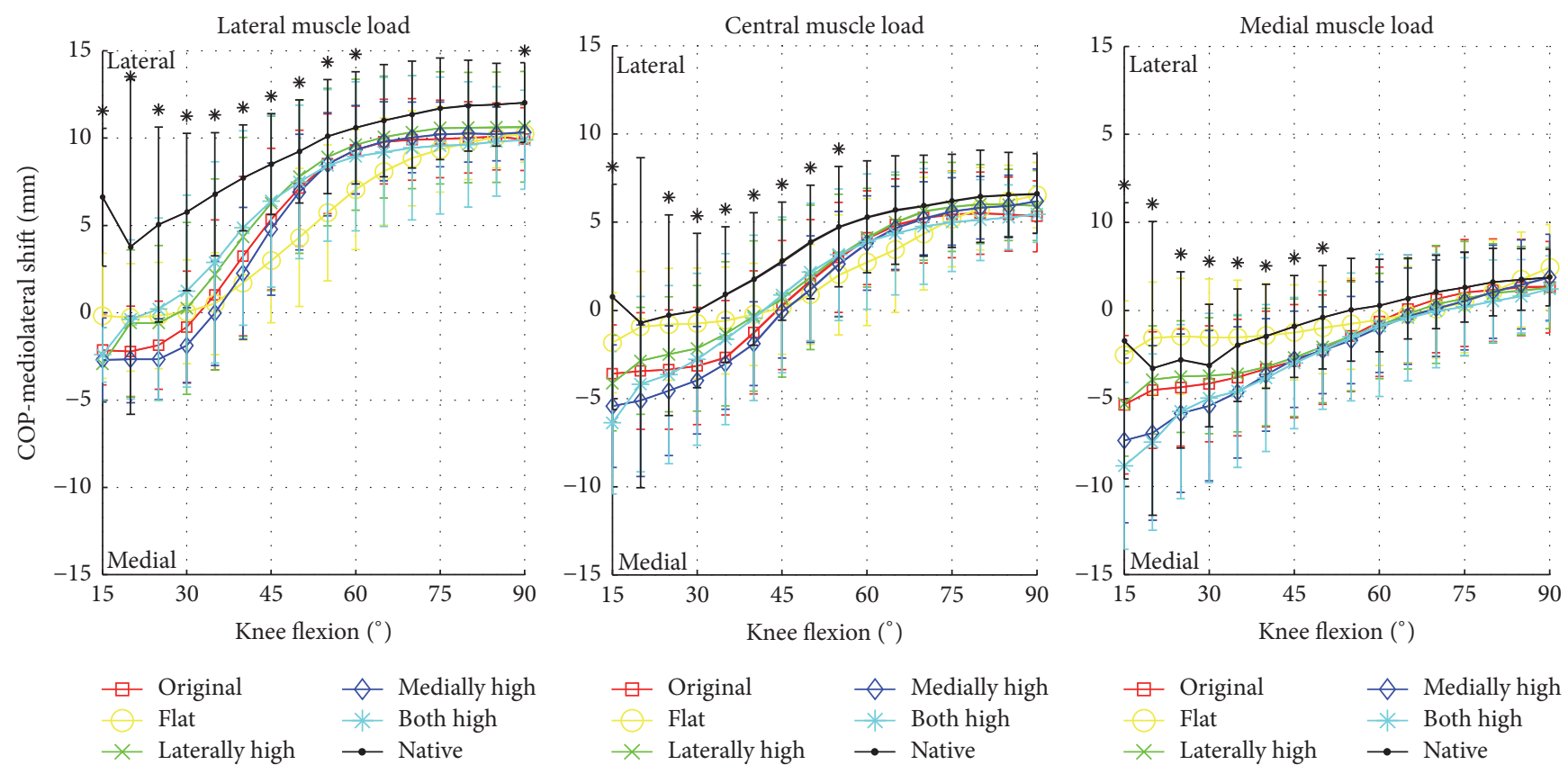

FIGURE 7: Medial and/or lateral displacement of the centre of pressure of the patella with increased lateral, central, and increased medial muscle load after implantation of the different design variations without patellar resurfacing (native, original, flat, laterally high, medially high, and both sides high) ( ${ }^{*}$ significant difference in ANOVA). 

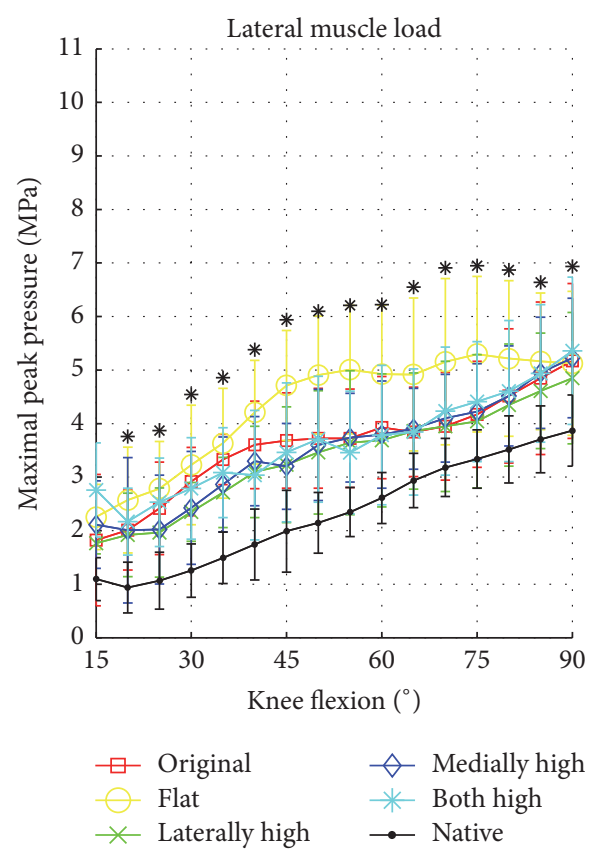
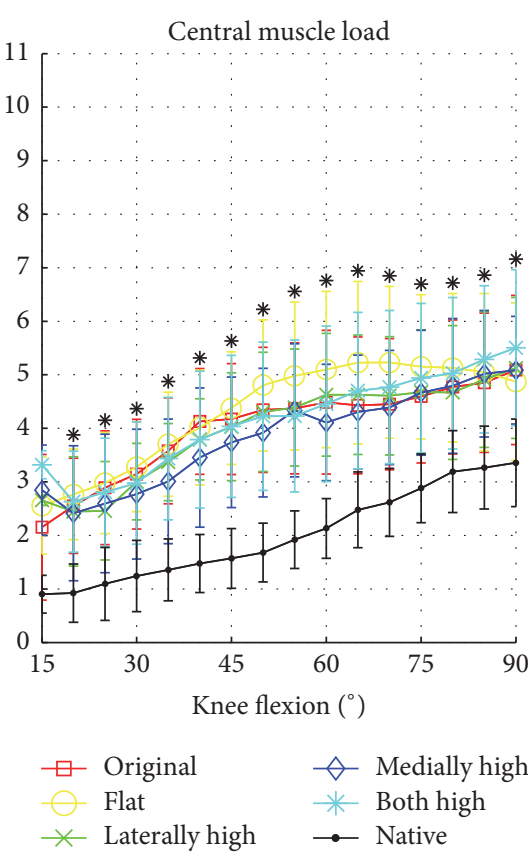
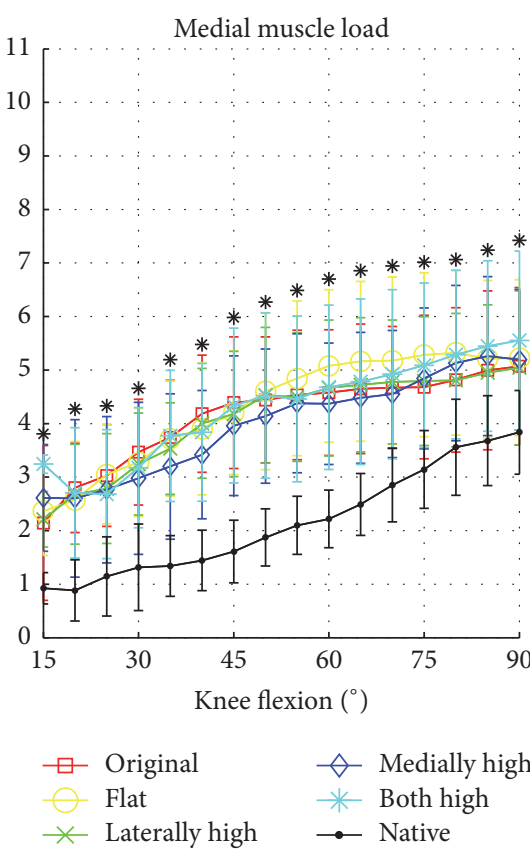

FIGURE 8: Change in maximum pressure with increased lateral, central, and increased medial muscle load after implementation of different design variations with patellar resurfacing (native, original, flat, laterally high, medially high, and both sides high) $\left({ }^{*}\right.$ significant difference).

the maximum patellofemoral pressure increased slightly, compared to native specimen, after implantation of the femoral and tibial prosthesis, but considerably after additional patellar resurfacing. These results are consistent with observations from our own preliminary work on original Genesis II implants [25]. Other authors have also described influences of the implantation of total knee endoprostheses on patellofemoral pressures $[24,26,28,37]$ but delivered sometimes controversial results. It is difficult to compare the various studies, as they differ not only with regard to the prosthesis systems tested, but also with regard to the experimental settings and measuring methods used. The work groups of Takeuchi et al. [30] and Tanzer et al. [16] each tested different implant systems on the same experimental setting. Because these implant systems exhibit considerable differences in their overall design, however, it was still difficult to impossible to verify the specific influence of the respective patellofemoral groove.

The purpose of this study was therefore to analyse the influence of groove variations on patellofemoral kinematics as well as patellofemoral pressure through selective modification of the femoral patellar groove of the established standard prosthesis of Genesis II type. These data should help further optimise the femoral patella groove design of total knee endoprostheses.

In the analysis of the patellofemoral kinematics, the different design variations, including the flat groove design, had on average no apparent influence on the alignment of the patella in craniocaudal and anteroposterior orientation. There was a detectable effect on the mediolateral tilt and translation, however. The direct relationship between groove design and patellar motion could be seen here, especially in the tilting - the higher the lateral margin of the groove, the less the lateral tilting of the patella. Only in the case of the flat groove design was there a noticeable deviation from the rest of the designs. A significantly increased lateral tilting and a slight medial translation of the patella were detectable, which indicates that a certain orientation of the patella contributes to the improvement of the kinematics by increasing primarily the lateral margin of the femoral patellar groove. Yoshii et al. already tested two different prosthesis designs that differed mainly in the lateral elevation of the patellofemoral groove as well as the depth of the trochlear groove [38] and found considerable influence on the mediolateral tilting and shifting of the patella, if the likewise used patellar button was attached slightly medialised at the same time, which in a majority of patellae (Wibeeg types 2 and 3 [39]) corresponds to anatomical positioning. Tanzer et al. were also able to identify variations on the mediolateral patellar tracking in an in vitro study of prosthesis models of various manufacturers [16] but were unable to trace those effects back to the specific design parameters because of the generally different prosthesis design.

Considering the pressure distribution pattern of the different design variations, especially when comparing the original design with the flat as well as the both sides elevated/deeply recessed design, one notices that-after the native knee joint-the original design of Genesis II endoprosthesis exhibits the most extensive patellofemoral contact area. The flat profile has a smaller central contact area and the deeply recessed design has a contact area that is shifted more onto the condyles. Additional patellar resurfacing reduced the contact area significantly, to the point of an almost only punctual contact. In the individual specimens, 
the asymmetrical design variations produced very different pressure distribution patterns.

With regard to the peak pressures measured, the original, medially elevated, laterally elevated, and deeply recessed designs - without additional patellar resurfacing-produced on average similar results, whereas the flat design attracted attention with elevated peak pressures at low flexion angles up to approximately $50^{\circ}$. After additional patellar resurfacing as well, the flat groove profile exhibited considerably higher maximum pressures-but now in the flexion range between approximately $40^{\circ}$ and $80^{\circ}$-particularly with increased lateral muscle load, compared to the other designs. This once again proves the need for certain tracking of the patella. The results of the individual specimens (Table 1) in particular show as well that too much elevation of the lateral margin often leads to a pressure increase and is therefore also not recommendable.

Despite the on average similar results for most design variations, in terms of patellofemoral pressure distribution, large interindividual differences between the different designs were determined (Table 1). In the comparison of the individual specimens, the different designs even sometimes had an opposite effect on the pressure profile, which indicates that the different groove designs did not fit equally with the different specimens. This can probably be explained by the great differences of the native anatomy and substantiates the results from several finite element studies by Fitzpatrick et al. $[40,41]$. Among other things they reported that in the area of the patellofemoral groove in particular patient-specific influencing factors dominate [40]. In another study they were able to show that both the patellofemoral kinematics and the contact characteristics could be improved considerably after performance of total knee arthroplasty without patellar resurfacing if the prosthesis design was adapted to the native anatomy [41]. The fact that the conformity of the prosthesis with the patellar button $[29,42]$ and native patella [42] plays a major role with regard to the contact characteristics has already been shown in studies, which were reduced to this effect by application of static force by means of a material testing machine [29] or overlays of CAD models [42] at various flexion angles. In vitro studies using human specimens with prosthesis models of various manufacturers without [16] and with patellar resurfacing [30] also substantiated the large differences with regard to patellofemoral pressure distribution in various prosthesis designs, whereby it was once again difficult here to trace these differences back to actual design features because of the multifactorial influences, as already with the case with the kinematics.

Particularly, relating to the native femoral patellar groove, there are various, often greatly diverging norm variants based on the osseous form of the distal femur and patellofemoral surface. Taking into consideration our results and the available literature, it is at least questionable if these different shapes of the patella and patellar groove can be properly and adequately treated with just one standard prosthesis available only in different sizes (which until now has been the standard in clinical practice), in view of the various biomechanical circumstances. One possible approach to a solution here could be a prosthesis system that provides variations that differ only with regard to the shape of the patellofemoral groove, with the basic design remaining constant. For reasonable clinical use, hard clinical and/or radiological criteria would first need to be devised and established which enable a meaningful assignment of the individual design variations to the respective anatomical situation. In our view, an important parameter to be considered is the shape of the native patella bone, which can be classified according to the Wiberg classification [39].

\section{Conclusions}

In summary, the shape of the patellofemoral groove has a demonstrable influence on patellofemoral kinematics as well as patellofemoral pressure. This effect becomes apparent especially with different force distribution patterns on the quadriceps muscle. The greatest influence observed was on the patellar tilting, followed by the mediolateral shift of the patella. In our view, in the design particularly the slight elevation of the lateral margin of the groove is critical for counteracting the main effect-the lateral tilting of the patella-and improving the mediolateral tracking. However, a smooth contouring like that with the original design of Genesis II endoprosthesis suffices, which the results for patellofemoral pressure distribution also substantiate. Very large interindividual differences were also seen, especially with regard to the patellofemoral contact characteristics, which suggest the need for a greater selection option of prosthesis designs. Further studies are necessary, however, in order to be able to determine possible design variations and, above all, define hard criteria for their areas of use.

\section{Competing Interests}

This study was partly funded by Smith \& Nephew, but the design and execution of the experiments as well as the preparation of the manuscript were independently performed by the authors.

\section{Acknowledgments}

The authors gratefully acknowledge the financial support of Smith \& Nephew. They further acknowledge Elsevier language services for the English editing and translation of the article.

\section{References}

[1] L. G. Shiers, "Arthroplasty of the knee; preliminary report of new method," The Journal of Bone and Joint Surgery. British Volume, vol. 36, no. 4, pp. 553-560, 1954.

[2] German Hospital Quality Report 2013, Institute for Applied Quality Improvement and Research in Health Care, 2015.

[3] S. Li, Y. Chen, W. Su, J. Zhao, S. He, and X. Luo, "Systematic review of patellar resurfacing in total knee arthroplasty," International Orthopaedics, vol. 35, no. 3, pp. 305-316, 2011.

[4] R. Michalik, B. Rath, H.-R. Springorum, C. Lüring, and M. Tingart, "Anterior knee pain after total knee arthroplasty : 
causes, diagnosis and treatment," Orthopade, vol. 45, no. 5, pp. 386-398, 2016.

[5] W. Petersen, I. V. Rembitzki, G.-P. Brüggemann et al., "Anterior knee pain after total knee arthroplasty: a narrative review," International Orthopaedics, vol. 38, no. 2, pp. 319-328, 2014.

[6] D. Shervin, K. Pratt, T. Healey et al., "Anterior knee pain following primary total knee arthroplasty," World Journal of Orthopedics, vol. 6, no. 10, pp. 795-803, 2015.

[7] J. D. Swan, J. D. Stoney, K. Lim, M. M. Dowsey, and P. F. M. Choong, "The need for patellar resurfacing in total knee arthroplasty: a literature review," ANZ Journal of Surgery, vol. 80, no. 4, pp. 223-233, 2010.

[8] R. L. Barrack, T. Schrader, A. J. Bertot, M. W. Wolfe, and L. Myers, "Component rotation and anterior knee pain after total knee arthroplasty," Clinical Orthopaedics and Related Research, no. 392, pp. 46-55, 2001.

[9] C. Verlinden, P. Uvin, L. Labey, J. P. Luyckx, J. Bellemans, and H. Vandenneucker, "The influence of malrotation of the femoral component in total knee replacement on the mechanics of patellofemoral contact during gait: an in vitro biomechanical study," Journal of Bone and Joint Surgery-Series B, vol. 92, no. 5, pp. 737-742, 2010.

[10] C. König, A. Sharenkov, G. Matziolis et al., "Joint line elevation in revision TKA leads to increased patellofemoral contact forces," Journal of Orthopaedic Research, vol. 28, no. 1, pp. 1-5, 2010.

[11] D. N. Bracey, M. L. Brown, H. R. Beard et al., "Effects of patellofemoral overstuffing on knee flexion and patellar kinematics following total knee arthroplasty: a cadaveric study," International Orthopaedics, vol. 39, no. 9, pp. 1715-1722, 2015.

[12] K. C. Bertin and W. W. S. Lloyd, "Effect of total knee prosthesis design on patellar tracking and need for lateral retinacular release," Journal of Arthroplasty, vol. 28, no. 5, pp. 772-777, 2013.

[13] D. D. D’Lima, P. C. Chen, M. A. Kester, and C. W. Colwell Jr., "Impact of patellofemoral design on patellofemoral forces and polyethylene stresses," Journal of Bone and Joint Surgery A, vol. 85, supplement 4, pp. 85-93, 2003.

[14] P. F. Indelli, M. Marcucci, G. Pipino, S. Charlton, C. Carulli, and M. Innocenti, "The effects of femoral component design on the patello-femoral joint in a PS total knee arthroplasty," Archives of Orthopaedic and Trauma Surgery, vol. 134, no. 1, pp. 59-64, 2014.

[15] C.-G. Kong, S.-W. Park, H. Yang, and Y. In, "The effect of femoral component design on patellar tracking in total knee arthroplasty: genesis II prosthesis versus Vanguard prosthesis," Archives of Orthopaedic and Trauma Surgery, vol. 134, no. 4, pp. 571-576, 2014.

[16] M. Tanzer, C. A. McLean, E. Laxer, J. Casey, and A. M. Ahmed, "Effect of femoral component designs on the contact and tracking characteristics of the unresurfaced patella in total knee arthroplasty," Canadian Journal of Surgery, vol. 44, no. 2, pp. 127-133, 2001.

[17] S. M. Theiss, K. J. Kitziger, P. S. Lotke, and P. A. Lotke, "Component design affecting patellofemoral complications after total knee arthroplasty," Clinical Orthopaedics and Related Research, no. 326, pp. 183-187, 1996.

[18] A. Takahashi, H. Sano, M. Ohnuma et al., "Patellar morphology and femoral component geometry influence patellofemoral contact stress in total knee arthroplasty without patellar resurfacing," Knee Surgery, Sports Traumatology, Arthroscopy, vol. 20, no. 9, pp. 1787-1795, 2012.
[19] M. Saffarini, S. Zaffagnini, S. Bignozzi, F. Colle, M. Marcacci, and D. Dejour, "Does patellofemoral geometry in TKA affect patellar position in mid-flexion?" Knee Surgery, Sports Traumatology, Arthroscopy, vol. 23, no. 6, article 3565, pp. 1799-1807, 2015.

[20] H. Kainz, W. Reng, P. Augat, and S. Wurm, "Influence of total knee arthroplasty on patellar kinematics and contact characteristics," International Orthopaedics, vol. 36, no. 1, pp. 73-78, 2012.

[21] A. M. Merican, K. M. Ghosh, F. Iranpour, D. J. Deehan, and A. A. Amis, "The effect of femoral component rotation on the kinematics of the tibiofemoral and patellofemoral joints after total knee arthroplasty," Knee Surgery, Sports Traumatology, Arthroscopy, vol. 19, no. 9, pp. 1479-1487, 2011.

[22] S. Ostermeier, O. Buhrmester, C. Hurschler, and C. StukenborgColsman, "Dynamic in vitro measurement of patellar movement after total knee arthroplasty: an in vitro study," BMC Musculoskeletal Disorders, vol. 6, article 30, 2005.

[23] C. Browne, J. C. Hermida, A. Bergula, C. W. Colwell Jr., and D. D. D'Lima, "Patellofemoral forces after total knee arthroplasty: effect of extensor moment arm," Knee, vol. 12, no. 2, pp. 81-88, 2005.

[24] S. Fuchs, G. Schütte, H. Witte, and D. Rosenbaum, "Retropatellar contact characteristics in total knee arthroplasty with and without patellar resurfacing," International Orthopaedics, vol. 24, no. 4, pp. 191-193, 2000.

[25] U. G. Leichtle, M. Wünschel, C. I. Leichtle et al., "Increased patellofemoral pressure after TKA: an in vitro study," Knee Surgery, Sports Traumatology, Arthroscopy, vol. 22, no. 3, pp. 500-508, 2014.

[26] S. Matsuda, T. Ishinishi, S. E. White, and L. A. Whiteside, "Patellofemoral joint after total knee arthroplasty. Effect on contact area and contact stress," The Journal of Arthroplasty, vol. 12, no. 7, pp. 790-797, 1997.

[27] A. Steinbrück, C. Schröder, M. Woiczinski, A. Fottner, P. E. Müller, and V. Jansson, "Patellofemoral contact patterns before and after total knee arthroplasty: an in vitro measurement," BioMedical Engineering Online, vol. 12, article 58, 2013.

[28] C. Stukenborg-Colsman, S. Ostermeier, O. Burmester, and C. J. Wirth, "Dynamic in vitro measurement of retropatellar pressure after knee arthroplasty," Orthopade, vol. 32, no. 4, pp. 319-322, 2003.

[29] J. A. Szivek, P. L. Anderson, and J. B. Benjamin, "Average and peak contact stress distribution evaluation of total knee arthroplasties," Journal of Arthroplasty, vol. 11, no. 8, pp. 952963, 1996.

[30] T. Takeuchi, V. K. Lathi, A. M. Khan, and W. C. Hayes, "Patellofemoral contact pressures exceed the compressive yield strength of UHMWPE in total knee arthroplasties," The Journal of Arthroplasty, vol. 10, no. 3, pp. 363-368, 1995.

[31] M. Barink, H. Meijerink, N. Verdonschot, A. Van Kampen, and M. De Waal Malefijt, "Asymmetrical total knee arthroplasty does not improve patella tracking: a study without patella resurfacing," Knee Surgery, Sports Traumatology, Arthroscopy, vol. 15, no. 2, pp. 184-191, 2007.

[32] Y. Herzog, Assoziativ parametrisierte Entwicklung von Knieprothesen zur Herstellung im Sinterlithographie-Verfahren [M.S. thesis], University of Stuttgart, 2011.

[33] A. Lorenz, Y. Herzog, P. Schnauffer, M. Arnold, N. Wülker, and U. G. Leichtle, "Rapid prototyping-a promising technique for orthopaedic implant fabrication: application and validation 
of TKA prototypes for in-vitro testing," Computer Methods in Biomechanics and Biomedical Engineering, vol. 17, no. 1, pp. 5455, 2014.

[34] A. Lorenz, O. Müller, P. Kohler, M. Wünschel, N. Wülker, and U. G. Leichtle, "The influence of asymmetric quadriceps loading on patellar tracking - an in vitro study," Knee, vol. 19, no. 6, pp. 818-822, 2012.

[35] O. Müller, J. Lo, M. Wünschel, C. Obloh, and N. Wülker, "Simulation of force loaded knee movement in a newly developed in vitro knee simulator," Biomedical Engineering, vol. 54, no. 3, pp. 142-149, 2009.

[36] M. Wünschel, U. Leichtle, C. Obloh, N. Wülker, and O. Müller, "The effect of different quadriceps loading patterns on tibiofemoral joint kinematics and patellofemoral contact pressure during simulated partial weight-bearing knee flexion," Knee Surgery, Sports Traumatology, Arthroscopy, vol. 19, no. 7, pp. 1099-1106, 2011.

[37] S. Fuchs, G. Schütte, H. Witte, and D. Rosenbaum, "Welche retropatellaren veränderungen entstehen durch die implantation einer oberflächenkniegelenkprothese? Eine computersimulation," Unfallchirurg, vol. 103, no. 11, pp. 972-976, 2000.

[38] I. Yoshii, L. A. Whiteside, and Y. S. Anouchi, "The effect of patellar button placement and femoral component design on patellar tracking in total knee arthroplasty," Clinical Orthopaedics and Related Research, no. 275, pp. 211-219, 1992.

[39] G. Wibeeg, "Roentgenographs and anatomic studies on the femoropatellar joint: with special reference to chondromalacia patellae," Acta Orthopaedica, vol. 12, no. 1-4, pp. 319-410, 1941.

[40] C. K. Fitzpatrick, C. W. Clary, and P. J. Rullkoetter, "The role of patient, surgical, and implant design variation in total knee replacement performance," Journal of Biomechanics, vol. 45, no. 12, pp. 2092-2102, 2012.

[41] C. K. Fitzpatrick and P. J. Rullkoetter, "Influence of patellofemoral articular geometry and material on mechanics of the unresurfaced patella," Journal of Biomechanics, vol. 45, no. 11, pp. 1909-1915, 2012.

[42] H.-M. Ma, Y.-C. Lu, T.-G. Kwok, F. Y. Ho, C.-Y. Huang, and C.H. Huang, "The effect of the design of the femoral component on the conformity of the patellofemoral joint in total knee replacement," Journal of Bone and Joint Surgery B, vol. 89, no. 3, pp. 408-412, 2007. 


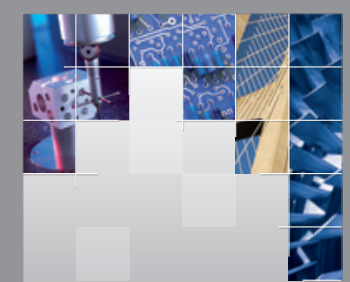

\section{Enfincering}
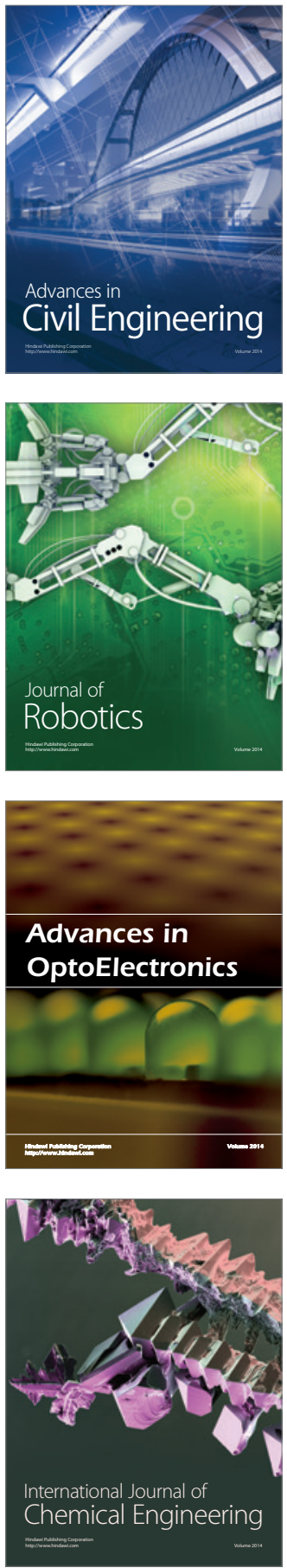

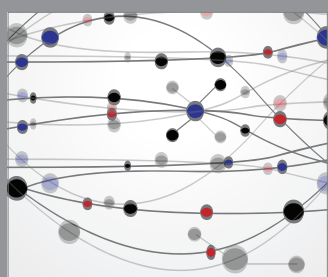

The Scientific World Journal

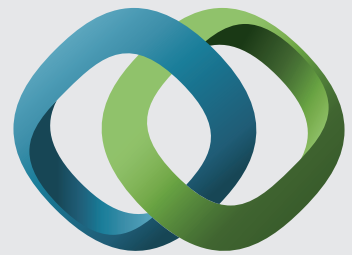

\section{Hindawi}

Submit your manuscripts at

https://www.hindawi.com
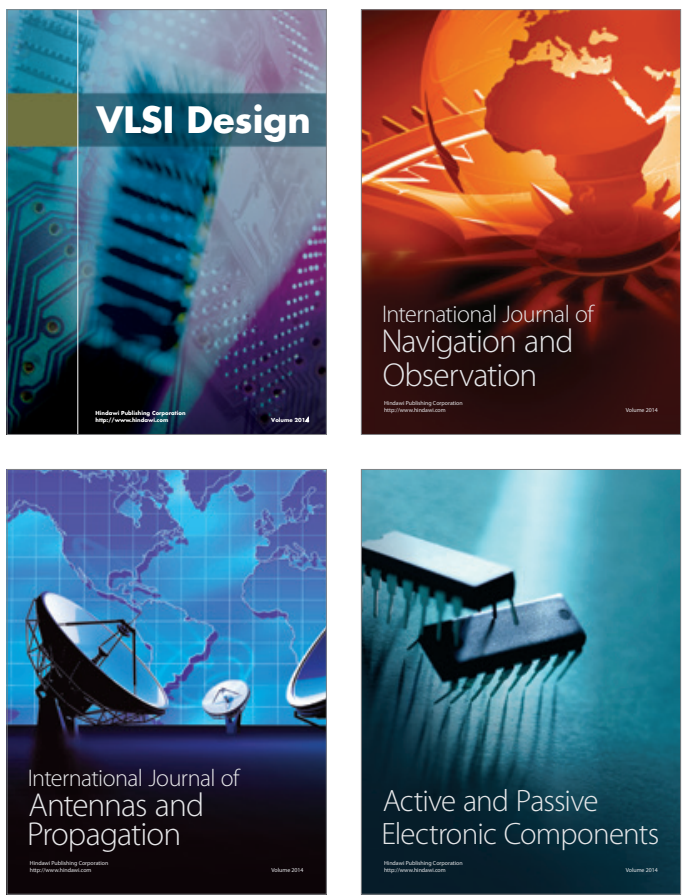
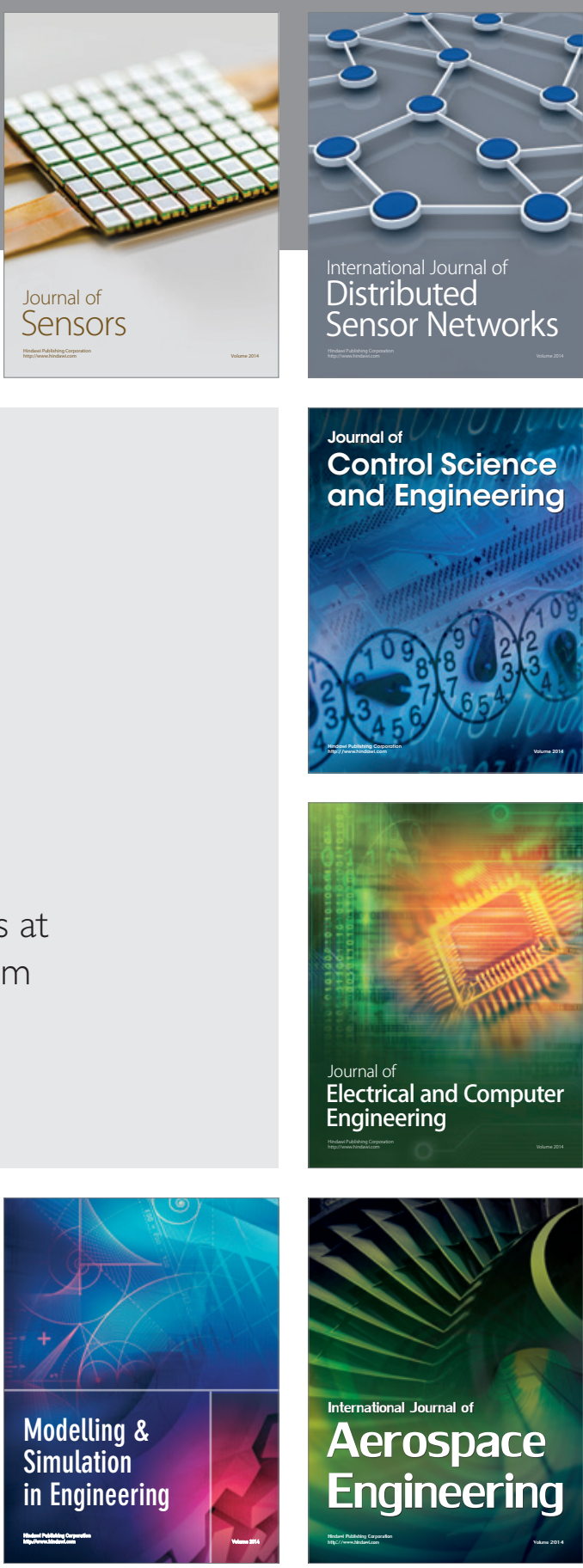

International Journal of

Distributed

Sensor Networks

$-$

Joumal of

Control Science

and Engineering
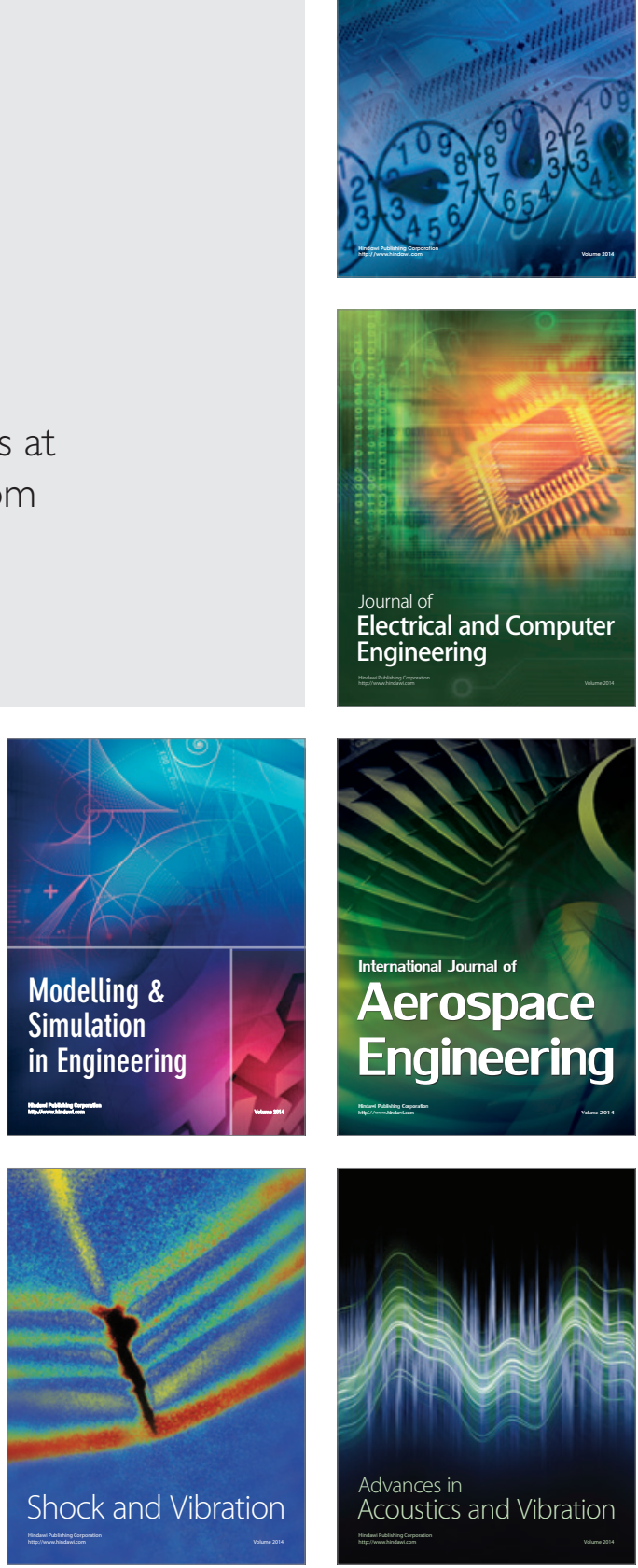